\title{
Maduración y comportamiento poscosecha de la feijoa (Acca sellowiana (0. Berg) Burret). Una revisión
}

\section{Ripening and postharvest behavior in the pineapple guava (Acca sellowiana (0. Berg) Burret). A review}

ALFONSO PARRA C. ${ }^{1,3}$

GERHARD FISCHER ${ }^{2}$

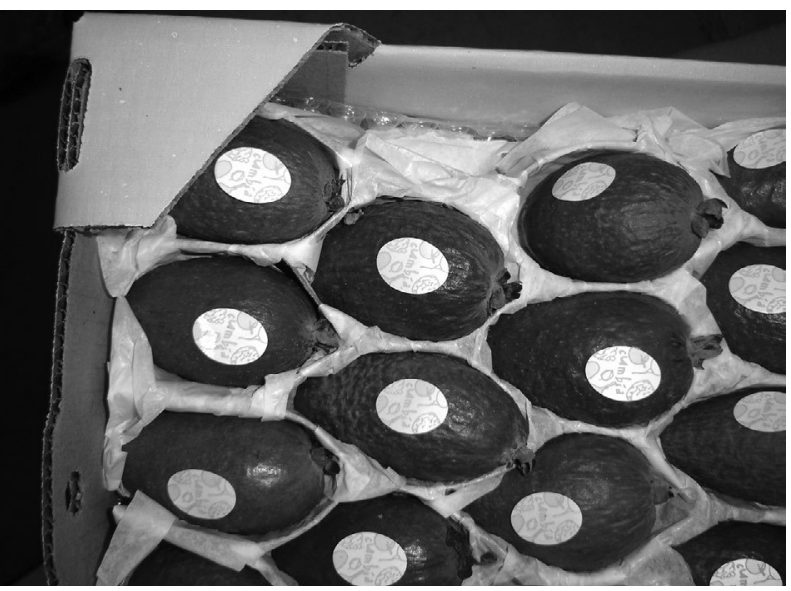

\section{RESUMEN}

La feijoa es considerada un cultivo promisorio en Colombia, por su potencial de exportación, características nutracéuticas y uso agroindustrial. Los diferentes estudios poscosecha indican que los frutos de feijoa presentan comportamiento típico climatérico, con elevadas tasas respiratorias y de producción de etileno, requiriendo refrigeración poscosecha inmediata para preservar su calidad (temperatura de 4 a $10^{\circ} \mathrm{C}$; humedad relativa de $90-95 \%$ ). A medida que transcurre el periodo poscosecha, aumentan la pérdida de peso y el $\mathrm{pH}$, y disminuye la firmeza del fruto. Los resultados son contradictorios en cuanto a la tendencia de los sólidos solubles y de la acidez titulable, influenciados probablemente por las características varietales, edad de la planta y por las condiciones climáticas y de cultivo a que hayan estado expuestos.

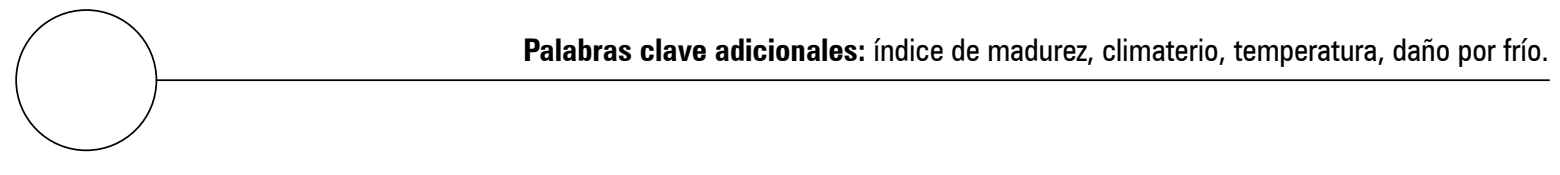

\footnotetext{
Facultad de Agronomía, Programa de Doctorado en Ciencias Agropecuarias, Área Agraria, Línea Fisiología de Cultivos; Facultad de Ingeniería, Departamento de Ingeniería Civil y Agrícola, Universidad Nacional de Colombia, Bogotá (Colombia).

2 Facultad de Agronomía, Departamento de Agronomía, Universidad Nacional de Colombia, Bogotá (Colombia).

3 Autor para correspondencia. aparrac@unal.edu.co
} 


\section{ABSTRACT}

Due to its export potential, nutraceutical characteristics and agro-industrial use, the pineapple guava is considered a promising crop in Colombia. Different postharvest studies have indicated that the fruit has a typical climacteric behavior with high respiration rates and ethylene production, requiring immediate postharvest refrigeration to preserve its quality (temperatures of $4-10^{\circ} \mathrm{C}, 90-95 \%$ relative humidity). As the postharvest period elapses, fruit weight loss and $\mathrm{pH}$ increase; and fruit firmness decreases. The results are contradictory for the tendency of soluble solids and titratable acidity, probably influenced by varietal characteristics, plant age and climatic and growing conditions.

Additional key words: maturity index, climacteric, temperature, chilling.

Fecha de recepción: 04-02-2013

Aprobada para publicación: 28-05-2013

INTRODUCCIÓN

La familia Myrtaceae está representada por aproximadamente 133 géneros y 3.800 especies (Wilson et al., 2001). La feijoa (Acca sellowiana (O. Berg) Burret) es originaria de Suramérica, en las zonas comprendidas entre el sur de Brasil, Uruguay, las partes altas del lado occidental de Paraguay y el nororiente de Argentina (Schumann y Lüdders, 1992; CCI, 2008); es una especie perenne y longeva, con alta capacidad de adaptación a diferentes zonas climáticas (Fischer, 2003). En condiciones subtropicales y estacionales presenta una cosecha anual, mientras que en zonas tropicales puede producir en forma continua durante todo el año (Quintero, 2012). La feijoa se considera un cultivo promisorio para la región andina colombiana, gracias a su excelente adaptación en las zonas comprendidas entre 1.800 y $2.700 \mathrm{~m}$ de altitud. Del fruto se pueden obtener más de 15 productos derivados de muy buena calidad (Quintero, 2012), tales como dulces, helados, postres, concentrados, sabajones, aperitivo vínico y otras bebidas.

Actualmente la producción comercial significativa de feijoa se limita a Nueva Zelanda, Georgia, Azerbaiyán, Colombia y California, aunque existe gran interés en establecer la producción comercial en Uruguay y Brasil (Dos Santos et al., 2009). La producción en las zonas con estaciones, particularmente en los países europeos, no es constante durante todo el año y solo en octubre y noviembre hay fruta disponible, reduciéndose notablemente su permanencia y reconocimiento en los mercados; esta condición representa una gran oportunidad para Colombia, que puede entrar al mercado europeo en la época de mayor demanda de productos frescos exóticos (noviembre a marzo) y durante los demás meses que presenten una demanda sostenida, razón por la cual la CCI (2008) ubica actualmente la feijoa "entre las 10 especies frutícolas agroindustriales promisorias en Colombia, pues se destaca por ser una fruta exótica, con gran potencial en los mercados extranjeros".

En Uruguay se conocen 11 cultivares, destacándose 'Botali' por su tamaño, su acentuado sabor y maduración tardía, así como 'M-4' de forma globosa, color amarillo rojizo y extraordinaria dulzura. En Brasil se tienen los cultivares Santa Elisa y Campineira. En Francia, la 'André' y 'Besson', de excelente calidad (Giacommetti y Lleras, 2012). En California se tienen los cultivares Apo1lo, Choiceana, Coolidge, Edenvale Improved 
Coolidge, Edenvale Late, Edenvale Supreme, Gemini, Mammoth, Moore, Nazemetz, Pineapple Gem, Trask, Triumph y Hehre (CRFG, 1996; Giacommetti y Lleras, 2012). En Colombia, el Centro Nacional de la Feijoa (Cenaf), ubicado en el municipio de La Vega en Cundinamarca, posee un banco de germoplasma conformado por cultivares procedentes de Nueva Zelanda y materiales establecidos en Colombia. El Cenaf ha priorizado la selección de cultivares adecuados a las condiciones climáticas de las diferentes regiones colombianas, para lo cual dispone de alrededor de 1.500 accesiones, entre las que se encuentran los cultivares Mammoth, Apollo, Gemini, Triumph, Coolidge, Unique y Marion (Perea et al., 2010) y los clones 41 (Quimba), 8-4 y 15-1 (Fischer, 2003). Según Rivas (2004), como resultado de las evaluaciones realizadas en el Cenaf, se cuenta con ocho variedades de feijoa: Triumph, Mammoth, Apollo, Gemini, Unique, Niza, Rionegro y Tibasosa, así como con seis clones sobresalientes: Marion, 15-1, 9-3, UN, Caldas Colombia y 41 (Quimba).

Los cultivos de feijoa en Colombia son establecidos a partir de diferentes variedades, lo cual se considera un factor importante para la polinización cruzada y obtención de frutos de buena calidad. Durante el año 2010 se cosecharon en Colombia 147 ha de feijoa de todas las variedades, con una producción total de 1.400 t, siendo los principales departamentos productores Boyacá, Caldas, Cundinamarca, Santander y Norte de Santander (Agronet, 2012). El color verde que tienen los frutos de las variedades de feijoa conocidas en Colombia, es considerado como un inconveniente para su comercialización, por ser poco atractivo; por eso se buscan cultivares de color amarillo y rojo (Giacommetti y Lleras, 2012).

Desde el punto de vista nutricional, el fruto de feijoa se caracteriza por su alto contenido de yodo y vitamina C (Hoffmann et al., 1994). La feijoa posee características inmunológicas y antioxidantes, gracias a que es un fruto rico en bioflavonoides, polifenoles activos como la catequina, leucoantocianinas, flavonoides, proantocianidinas y naftoquinonas (Ebrahimzadeh et al., 2008); tiene propiedades anticancerígenas e influye en la secreción de citoquinas en el intestino (Nakashima, 2001; Manabe e Inobe, 2005; Bontempo et al., 2007). El fruto tiene actividad antimicrobiana, que junto con las propiedades antioxidantes, permite considerarlo como materia prima para producción de nuevos medicamentos (Vuotto et al., 2000), que permitan prevenir, entre otras, enfermedades cardiovasculares, cáncer e infecciones. A nivel empírico, el uso de feijoa sobre heridas e infecciones en la piel acelera y mejora el proceso de cicatrización (Quintero, 2012). Además los frutos de feijoa son ricos en fibra, potasio, fósforo, magnesio, azúcar y calcio (Seymour et al., 1993; CCI, 2000; Schotsmans et al., 2011).

Durante la poscosecha se produce en los frutos una serie de cambios, tanto de síntesis como de degradación, que son controlados genéticamente y que conducen a la senescencia (Kader, 1992). Los cambios, aunque son muy variables entre las especies, incluyen generalmente la modificación de la ultraestructura y textura de la pared celular, así como cambios en: turgencia, jugosidad, conversión de almidones en azucares, incremento en la susceptibilidad a patógenos en la poscosecha, alteraciones en la biosíntesis de pigmentos y de compuestos responsables del sabor (Giovannoni, 2001); estos cambios son los que van a determinar la calidad poscosecha de los frutos.

Después de ser cosechadas, las feijoas maduran desde adentro hacia afuera; la fruta demasiado madura presenta pérdida de sabor (disminución de la concentración de sólidos solubles y acidez) y oscurecimiento de la pulpa y de las semillas (Thorp y Klein, 1987). Los cambios externos de calidad que se producen durante la maduración poscosecha no son dramáticos, lo que hace difícil determinar el grado de madurez de los frutos por medios visuales, el tacto u otras técnicas no 
destructivas, como clasificación por densidad (Clark et al., 2005), rigidez o impulsos acústicos (Gaddam et al., 2005).

Teniendo en cuenta el potencial de la feijoa en los mercados internacionales, la gran variedad de subproductos que se pueden elaborar con esta y la posibilidad de obtener nuevos medicamentos, el objetivo de esta revisión es conocer su maduración y comportamiento poscosecha, con el fin de poder dar un manejo adecuado de los frutos, encaminado a conservar su calidad, reduciendo perdidas durante la comercialización.

\section{MADURACIÓN EN PLANTA E ÍNDICES DE MADUREZ}

El grado de madurez apropiado para realizar la cosecha de la fruta es de gran importancia ya que repercute en su vida poscosecha así como en su comercialización. Es indispensable distinguir entre madurez fisiológica y madurez comercial. La madurez fisiológica es la que alcanza el fruto cuando está sujeto a la planta, alcanzando su máximo crecimiento e indica el momento oportuno de la recolección. La madurez comercial se refiere a las exigencias de calidad de un mercado en particular, las cuales dependen del uso que se le vaya a dar y el tiempo que dure su comercialización (Parra-Coronado y Hernández-Hernández, 2008).

La identificación del grado de madurez apropiado para realizar la cosecha de la fruta es difícil de establecer en las feijoas, produciéndose la maduración del fruto con cambios sutiles en la composición química, reducciones en la firmeza y el color de la piel (Clark et al., 2005), alcanzándose la madurez de recolección entre 120 y 150 d desde botones florales (Quintero, 2012), coincidiendo generalmente con la abscisión natural de la fruta (Thorp y Klein, 1987). La feijoa cosechada prematuramente no desarrolla completamente su sabor y textura característicos; dejar la fruta demasiado tiempo en el árbol dará lugar a pérdidas considerables por golpes como resultado de la caída natural de los frutos al suelo (Quintero, 2012).

Schotsmans et al. (2011) indican que existen importantes diferencias entre los cultivares, con respecto a la velocidad de maduración y a la caída natural de la fruta y describen los índices de madurez más utilizados para la feijoa de la siguiente forma: en la prueba de consistencia, se toma la fruta por su zona ecuatorial y si está suave, se considera apta para cosechar; con la prueba de abscisión (resistencia del pedúnculo) las laminillas medias y a menudo las paredes primarias de las células (que unen el pedúnculo al fruto) se gelifican, de manera que las células ya no están soldadas entre sí y se separan, produciéndose el desprendimiento del fruto.

Los cambios en la intensidad del color de la corteza, así como la forma y tamaño del fruto (según variedad), son otros índices de cosecha utilizados en feijoa; la capacidad de identificar variaciones en madurez/maduración es uno de los principales desafíos que inhibe la entrega de una calidad constante y por lo tanto, retarda el desarrollo de las industrias comerciales (Vela et al., 2009), haciéndose indispensable la implementación de técnicas no destructivas que permitan poner de manifiesto pequeñas variaciones en el grado de madurez de los frutos. En feijoa es común encontrar gran variabilidad en las tasas de maduración y comportamiento durante el almacenamiento, debido principalmente a la mezcla de diferentes grados de madurez y a las dificultades inherentes al momento de cosechar la fruta (Schotsmans et al., 2011).

\section{MADURACIÓN POSCOSECHA DEL FRUTO}

Según Amarante et al. (2008) las bases fisiológicas de los procesos de maduración de los fru- 
tos de feijoa durante la poscosecha no son bien conocidas, lo que hace difícil el establecimiento de estrategias de conservación de su calidad en poscosecha. Sin embargo, algunos estudios realizados permiten dilucidar algunas estrategias de conservación de la calidad en poscosecha, tal como se indica a continuación.

\section{Cambios fisiológicos}

Según Mercado-Silva et al. (1998), los cambios fisiológicos y químicos que se presentan en el proceso de maduración poscosecha de las frutas están influenciados por el estado de madurez en la cosecha y por las condiciones climáticas predominantes en el cultivo (época del año). En muchos frutos, el inicio de la maduración, a partir de la recolección, está acompañado por un aumento en la intensidad respiratoria y en la producción de etileno, alcanzando su máximo valor cuando el fruto está completamente maduro (madurez de consumo), y disminuyendo durante la senescencia; los frutos que presentan este comportamiento se les denomina climatéricos; los frutos no climatéricos no presentan aumento de la respiración (Kays, 1997; Pantástico et al., 1984).

El comportamiento fisiológico poscosecha de los productos hortofrutícolas está influenciado por sus condiciones de almacenamiento, siendo la temperatura de almacenamiento el principal factor (Parra-Coronado y Hernández-Hernández, 2008). La prolongación de la vida en almacenamiento de los productos agrícolas usando bajas temperaturas (lo más cerca posible del punto más bajo que el producto puede resistir sin comprometer su calidad), se basa en la disminución de su intensidad respiratoria, la cual a su vez, reduce la velocidad de la actividad enzimática (Wills et al., 1998). La velocidad de las reacciones enzimáticas se incrementa exponencialmente con el aumento de la temperatura. Se ha encontrado que por cada $10^{\circ} \mathrm{C}$ de aumento de la temperatura, los cambios fisiológicos y químicos se incrementan en un valor de 2 a 3 veces $\left(Q_{10}\right)$, siendo más drástico este aumento cuando se va de temperaturas muy bajas a temperaturas más altas (Kader, 1996).

\section{Intensidad respiratoria (IR) y producción de etileno}

Los estudios realizados indican que diferentes clones de feijoa de Brasil, Uruguay y Colombia ('Quimba' y '8-4') presentan un comportamiento típico climatérico, con variación de los valores de IR (entre 18,5 y 129,7 $\mathrm{mg} \mathrm{kg}^{-1} \mathrm{~h}^{-1}$ de $\mathrm{CO}_{2}$ ) y producción de etileno (entre 11,72 y $50,0 \mu \mathrm{L}$ $\mathrm{kg}^{-1} \mathrm{~h}^{-1}$ ) para temperaturas de almacenamiento de 4 y $23^{\circ} \mathrm{C}$ respectivamente (Seymour et al., 1993; Gallego-Corrales et al., 2003; Gálvis, 2003; Valderrama et al., 2005a; Rodríguez et al., 2006; Amarante et al., 2008; Velho et al., 2011). En los frutos almacenados a $4^{\circ} \mathrm{C}$ no se presentó pico climatérico, observándose gran disminución en la IR (Velho et al., 2011). En la feijoa, la producción de etileno es baja durante el periodo preclimatérico $\left(0,1-0,4 \mu \mathrm{L} \mathrm{kg}^{-1} \mathrm{~h}^{-1}\right.$ a $\left.20^{\circ} \mathrm{C}\right)$, la cual se incrementa considerablemente durante el periodo climatérico $\left(40-50 \mu \mathrm{L} \mathrm{kg}^{-1} \mathrm{~h}^{-1}\right.$ a $\left.20^{\circ} \mathrm{C}\right)$. La susceptibilidad de la feijoa al etileno es baja, y el efecto de este, es el de adelantar la maduración del fruto, produciendo variación en la tonalidad del color verde y ablandamiento de la pulpa, sin afectar el sabor (Seymour et al., 1993; Wills et al., 1998). La feijoa presenta alta producción de etileno comparativamente con otros frutos climatéricos.

Amarante et al. (2008) trabajaron con los genotipos Brasil (Acesso 387) y Uruguay (Acesso 454), almacenados a $0,5,10,20$ y $30^{\circ} \mathrm{C}$ (humedad relativa [HR] 90-95\%), encontrando que hubo incremento sustancial en la tasa respiratoria de ambos genotipos al ir aumentando la temperatura entre 0 y $30^{\circ} \mathrm{C}$, con coeficiente metabólico $\left(\mathrm{Q}_{10}\right)$ de 3,5 aproximadamente. Estos valores de $\mathrm{Q}_{10}$ son superiores a los observados en la misma franja de temperaturas para diversas especies de frutos $\left(\mathrm{Q}_{10}\right.$ de 2,3$)$, lo cual indica una elevada actividad metabólica de los frutos al incrementar la temperatura, lo que resulta en un menor periodo de almacenamiento y una rápida senes- 
cencia poscosecha (Wills et al., 1998). Debido a esto, los frutos de feijoa requieren cuidados especiales de manejo, particularmente con relación al enfriamiento en la preservación de su calidad poscosecha (Thorp y Klein, 1987; Hoffmann et al., 1994), debiéndose almacenar a las temperaturas mínimas tolerables por el fruto para que no se presenten daños por frio. Gallego-Corrales et al. (2003) determinaron el cociente respiratorio (CR) para tres temperaturas de almacenamiento (ambiente, 12 y $7^{\circ} \mathrm{C}$ ), encontrando que en todos los casos el CR fue superior a 1, lo que indica que el fruto de feijoa posiblemente utiliza ácidos orgánicos como sustrato respiratorio en el proceso de maduración en poscosecha (Wills et al., 1998).

\section{Transpiración (pérdida de peso)}

Según Kays y Paull (2004), la transpiración y en menor medida el consumo de sustratos, son la razón de la pérdida de peso de los frutos durante la maduración en poscosecha. La pérdida de agua libre es una de las principales causas de deterioro de los frutos, ya que se originan no solamente pérdidas cuantitativas directas, sino también de calidad relacionadas con el aspecto, pérdida de brillo, de turgencia, cambios en la textura y el valor nutricional (Kader, 1992). Las tasas reportadas de pérdida de peso para frutas empacadas en polietileno están en el rango de $0,75 \%$ (AlHarthy et al., 2008) a 1,25\% (Hoffmann et al., 1994) por semana en temperaturas de almacenamiento bajas $\left(0-4^{\circ} \mathrm{C}\right)$. La pérdida de peso semanal sin protección de bolsa de polietileno o en condiciones de almacenamiento a temperatura ambiente puede ser tan alta como el 5,5\% del peso inicial de la fruta (Hoffmann et al., 1994).

Rodríguez et al. (2006) encontraron que las pérdidas totales de peso para feijoas almacenadas a temperatura ambiente $\left(16,3^{\circ} \mathrm{C}, \mathrm{HR} 65,1 \%\right)$, son de $17,5 \%$ para el 'Clon 8-4' en $14 \mathrm{~d}$ de almacenamiento, y de 17,3\% en $18 \mathrm{~d}$ de almacenamiento para 'Quimba'. Estos resultados indican que el 'Clon 8-4' es más susceptible a las pérdidas de peso en condiciones de temperatura ambiente $y$ humedad relativa baja que 'Quimba', razón por la cual la fruta debe comercializarse antes del día 5 , en el cual presenta aún excelente calidad comercial. En el caso del clon Quimba, ellos afirman que los frutos se deben comercializar antes del día 7, en condiciones de la Sabana de Bogotá.

Rodriguez et al. (2006) señalan que en los frutos de feijoa la pérdida de peso está correlacionada directamente con la tasa respiratoria e inversamente con la acidez titulable, lo que indica que la pérdida de peso está asociada (entre otros) con los procesos oxidativos. Hoffmann et al. (1994) y Valderrama et al. (2005a) indican que en cuanto al efecto de la temperatura y de la humedad relativa sobre la pérdida de peso, la feijoa se puede conservar por largos periodos a bajas temperaturas observándose una pérdida de peso baja y una pérdida menor de sólidos solubles totales.

\section{Cambios físicos}

\section{Firmeza de la fruta}

Según Gálvis et al. (2002), la maduración poscosecha de algunas frutas se caracteriza por el ablandamiento de la pulpa, ocasionado por diferentes factores, entre ellos, la acción de las enzimas hidrolasas de la pared de la célula, las cuales actúan sobre la pectina; la enzima responsable de la solubilización de la pectina es la poligalacturonasa (PG), la cual presenta aumento en su actividad a medida que avanza la maduración. La actividad de la PG en la feijoa es mayor en el interior del mesocarpio, lo cual sugiere que el ablandamiento se inicia desde el interior hacia el exterior del mismo (Gálvis et al., 2001). Las celulasas también están relacionadas con el ablandamiento de la fruta. La actividad de la celulasa en el fruto verde es baja, pero se incrementa rápidamente durante la maduración (Kays, 1997). La dureza del fruto es una característica relevante desde el punto de vista del consumo y es un factor que debe tenerse en cuenta para el diseño de empaques y sistemas de transporte poscosecha del producto (Herrero y Guardia, 1992). 
Diferentes autores indican que la firmeza de la feijoa ('Quimba' y 'Clon 8-4'), al igual que en la mayoría de los frutos, decrece a medida que avanza el periodo poscosecha (Herrera y Quicazán, 2000; Rodriguez et al., 2006; Uyabán y Téllez, 2009). Herrera y Quicazán (2000) indican que varía desde $25 \mathrm{~kg}$-f en el momento de la cosecha, a aproximadamente $5 \mathrm{~kg}$-f 12 d después, mientras que Uyabán y Téllez (2009) reportan variaciones desde 11,34 a $3,40 \mathrm{~kg} \mathrm{~cm}^{-2}$, comportamiento que concuerda con lo encontrado por diferentes autores para guayabas (Mercado-Silva et al., 1998; Jacomino et al., 2003; Vieira et al., 2008; Solarte et al., 2010).

\section{Cambio de color}

Según Mercado-Silva et al. (1998), el cambio de color durante la poscosecha se presenta por degradación de la clorofila y por la síntesis de otros pigmentos como antocianinas y carotenoides. Hernández et al. (2007) indican que el color medido como ángulo Hue $\left({ }^{\circ} \mathrm{h}\right)$ representa el color o tonalidad, que varía de $0^{\circ}$ en color rojo puro a $180^{\circ}$ en color verde puro. En algunos cultivares de feijoa, disminuye el ángulo Hue $\left({ }^{\circ} \mathrm{h}\right)$, representando perdida de color verde (Velho et al., 2011); en otros cultivares, no es posible observar variaciones significativas en el color de la epidermis durante la maduración poscosecha de los frutos (East et al., 2009). Aumentando la temperatura se promueve la maduración y por ende la degradación de la clorofila y la reducción de ${ }^{\circ} \mathrm{h}$ de la epidermis en feijoa (Amarante et al., 2008), la cual no cambia de color debido a la genética de la fruta, variando solamente la tonalidad del verde.

\section{Cambios bioquímicos}

\section{Carbohidratos y sólidos solubles totales (SST)}

Los carbohidratos, en particular los azucares, son constituyentes muy importantes en las plantas ya que forman parte de la estructura de las células y funcionan como reservas de energía; algunos carbohidratos simples como sacarosa y fructosa se consideran atributos de calidad de algunos productos como las frutas (Solarte et al., 2010). La feijoa presenta altos niveles de almidón en el momento de la cosecha, el cual se hidroliza durante la maduración en poscosecha, produciendo aumento de los SST ('Brix). Según Rodríguez et al. (2006), los azúcares más abundantes durante el proceso de maduración de la feijoa son fructosa, sacarosa y glucosa.

En los frutos de feijoa, no hay consenso en cuanto a la variación de los SST durante la poscosecha; algunos autores indican que estos aumentan hasta el climaterio y luego disminuyen (Herrera y Quicazán, 2000; Rodriguez et al., 2006; Uyabán y Téllez, 2009), mientras que otros reportan que los SST disminuyen durante la poscosecha o permanecen constantes cuando el fruto se almacena a baja temperatura (Velho et al., 2011), con valores que oscilan entre 5 y $15^{\circ}$ Brix. Estos comportamientos disímiles, probablemente, estén influenciados por las características varietales, por la edad de la planta y por las condiciones climáticas y de cultivo a que hayan estado expuestos (Osterloh et al., 1996).

\section{Ácidos orgánicos y acidez total titulable (ATT)}

Los ácidos orgánicos desempeñan un papel crítico en el mantenimiento de la calidad (aroma y sabor característico de los frutos), en el valor nutricional de los alimentos y en el metabolismo poscosecha; algunos son componentes esenciales en el ciclo de los ácidos tricarboxílicos de la respiración y son una fuente de energía disponible que puede ser utilizada cuando otras fuentes se agotan (Solarte et al., 2010). Los principales ácidos presentes en la pulpa del fruto de feijoa son el ácido cítrico (9,84 g/100 g), el ácido málico (1,72 g/100 g) y el ácido succínico (0,49 g/100 g) (Herrera y Quicazán, 2000). El contenido de ácido ascórbico de la feijoa en el grado de madurez de consumo es de 28 mg/100 g (Herrera y Quicazán, 2000). 
Rodríguez et al. (2006) observaron que en frutos de feijoa la ATT aumenta hasta el climaterio y luego disminuye, mientras que otros autores, (Herrera y Quicazán, 2000; Uyabán y Téllez, 2009; Velho et al., 2011), reportan que la ATT disminuye durante el almacenamiento (4 a $23^{\circ} \mathrm{C}$ ), con valores de ATT que oscilan entre 0,3 y $4,3 \%$, comportamientos muy similares a los encontrados para SST. Rodríguez et al. (2006) indican que después del climaterio, los clones de feijoa, 8-4 y Quimba, presentan una correlación inversa entre la ATT y los niveles de SST, la pérdida de peso y la intensidad respiratoria, indicando que la acidez disminuye en el proceso de maduración porque en la respiración se degradan ácidos orgánicos (CR>1), confirmando lo encontrado por Gallego-Corrales et al. (2003).

\section{$p H$}

Cuando la fruta tiene fines industriales, el valor del pH es importante, ya que está relacionado con los costos de transformación del producto (Rodríguez et al., 2006). En los frutos 'Quimba' y 'Clon 8-4', Uyabán y Téllez (2009) indican que el pH aumenta de 2,8 a 3,05 a medida que avanza el periodo poscosecha, comportamiento similar al encontrado por Herrera y Quicazán (2000), quienes reportan aumento de $\mathrm{pH}$ de 3,1 a 3,5; el incremento del $\mathrm{pH}$ indica menores valores de ATT o disminución de esta a medida que transcurre el periodo poscosecha. Según Rodríguez et al. (2006), para los frutos de feijoa el pH también puede fluctuar según la variedad, las condiciones agroecológicas en las que se desarrolle el fruto y las condiciones de almacenamiento.

\section{Compuestos volátiles}

Durante la maduración poscosecha se producen ciertos compuestos volátiles, normalmente presentes en pequeñas cantidades, que son los responsables del aroma, constituyendo un factor organoléptico a considerar en la composición y calidad del fruto de feijoa, habiéndose identifi- cado más de 85 compuestos volátiles, siendo los principales: hidrocarbonos, alcoholes (constituyen alrededor del $50 \%$ en algunas variedades) y metilbenzoato (Cacioppo, 1982; Seymour et al., 1993). En la literatura consultada no se encontró información sobre los compuestos volátiles para las variedades cultivadas en Colombia.

\section{Patología y desordenes fisiológicos}

\section{Enfermedades}

Según Velho et al. (2011) las pudriciones, manchas superficiales y manchas deprimidas que se presentan en la epidermis de frutos de feijoa durante la poscosecha, son directamente proporcionales a la temperatura de almacenamiento (mayor a $23^{\circ} \mathrm{C}$ que a $4^{\circ} \mathrm{C}$, por $15 \mathrm{~d}$ ); la pudrición de los frutos está asociada a una reducción significativa de la ATT. Las manchas superficiales y deprimidas observadas en las feijoas producidas en Brasil son semejantes a las observadas en feijoas producidas en Nueva Zelanda (Thorp y Bieleski, 2002). Aparentemente, esos desordenes pueden ser causados por daños mecánicos durante la cosecha y el manejo poscosecha y se expresan durante la maduración y senescencia (Thorp y Bieleski, 2002), haciéndose necesario implementar operaciones de manejo poscosecha que minimicen este tipo de daños (empaques adecuados, manejo cuidadoso, etc.). Las pudriciones se desarrollan después del almacenamiento refrigerado, cuando los frutos son mantenidos a temperatura ambiente (East et al., 2009).

El potencial de almacenaje de feijoas a $23^{\circ} \mathrm{C}$ es inferior a una semana (limitado principalmente por oscurecimiento de la pulpa, manchas en la epidermis y pudriciones) y a $4^{\circ} \mathrm{C}$ es inferior a $30 \mathrm{~d}$, limitado principalmente por el oscurecimiento de la pulpa (Velho et al., 2011). Para feijoas almacenadas a temperatura ambiente $\left(16,3^{\circ} \mathrm{C}\right.$; HR $\left.65,1 \%\right)$, el pardeamiento de la pulpa se presentó a partir del día 8 para el 'Clon 8-4', mientras que para 'Quimba' se presentó a partir del día 16 (Rodríguez et al., 2006). 


\section{Daño por frío}

Velho et al. (2011) encontraron que el rápido deterioro de la calidad de feijoa después de la cosecha fue ocasionada por el desarrollo acentuado de tres trastornos: (1) manchas oscuras superficiales en la epidermis, acompañadas por numerosos puntos oscuros; (2) manchas oscuras levemente deprimidas en la epidermis, principalmente en la región mediana de los frutos, y (3) oscurecimiento de la pulpa. En este estudio no hubo presencia de patógenos en las manchas oscuras superficiales y levemente deprimidas en la epidermis, mostrando que estos síntomas corresponden a un trastorno fisiológico y el oscurecimiento de la pulpa aumentó acentuadamente, tanto en los frutos almacenados a $23^{\circ} \mathrm{C}$, como en los frutos almacenados a $4^{\circ} \mathrm{C}$. Este trastorno puede ser ocasionado principalmente por aumento de la actividad de la enzima polifenoloxidasa (PPO) o por la degradación de azucares en el fruto, e intensificado por altas temperaturas y baja HR durante el almacenamiento (Thorp y Bieleski, 2002). En algunos casos, el oscurecimiento de la pulpa de la feijoa puede ser causado por la excesiva reducción de los niveles de $\mathrm{O}_{2} \mathrm{O}$ aumento de los niveles de $\mathrm{CO}_{2}$ en el ambiente de almacenamiento (East et al., 2009), y como resultado de largos periodos de almacenamiento a $4^{\circ} \mathrm{C}$, ocasionando así daño por frío (Thorp y Bieleski, 2002).

La feijoa presenta daños por frío al almacenarse a temperaturas menores a $5^{\circ} \mathrm{C}$; en los genotipos de feijoa del tipo Uruguay, cultivados en Nueva Zelanda, se han reportado daño por frío a $0^{\circ} \mathrm{C}$, recomendándose por lo tanto el almacenamiento a $4^{\circ} \mathrm{C}$ (Thorp \& Klein, 1987). Sin embargo, en los frutos de feijoa cosechados en el sur de Brasil y almacenados a 0 y $2^{\circ} \mathrm{C}$ por $28 \mathrm{~d}$, seguidos de $2 \mathrm{~d}$ a temperatura ambiente, no hubo daño por frío (Hoffmann et al., 1994). Los trabajos realizados parecen indicar que los frutos del tipo Brasil son menos sensibles al daño por frío que los del tipo Uruguay, cuando se almacenan a temperaturas cercanas a $0^{\circ} \mathrm{C}$ (Amarante et al., 2008). De igual manera, con los resultados obtenidos por Valderrama et al. (2005a) se puede afirmar que la fruta soporta almacenamientos con temperatura cercana a $1^{\circ} \mathrm{C}$.

En Colombia, el rango de temperatura de almacenamiento recomendada para feijoa está entre 5 y $10^{\circ} \mathrm{C}$ (Gálvis, 2001), mientras que una temperatura más baja de $4,5^{\circ} \mathrm{C}$ se utiliza en Nueva Zelanda. A $4^{\circ} \mathrm{C}$, los principales cultivares comerciales de Nueva Zelanda (Apollo, Géminis, Opal Star, Pounamu y Triumph) tienen en general una vida de almacenamiento comercial de cuatro semanas, con un máximo de $5 \mathrm{~d}$ de vida útil posterior a $20^{\circ} \mathrm{C}$, antes de que la fruta se vuelva inaceptable en términos de sabor y pardeamiento interno de la pulpa; los frutos de 'Unique' tienen una gran reducción de la vida de almacenamiento, desarrollándose el pardeamiento de la pulpa después de $7 \mathrm{~d}$; la HR recomendada es del 90 al 95\% en almacenes refrigerados para evitar la pérdida de agua durante el almacenamiento (Thorp y Bieleski, 2002).

\section{Algunos tratamientos para prolongar vida útil}

Se ha demostrado que el tratamiento más efectivo para prolongar la vida útil poscosecha de productos agrícolas es el almacenamiento a baja temperatura (Parra-Coronado y HernándezHernández, 2008). Se han utilizado diferentes técnicas buscando conservar la calidad de los productos durante un mayor tiempo en poscosecha, tales como el almacenamiento en atmósferas controladas o modificadas (Herrero y Guardia, 1992; Seymour et al., 1993; Hoffmann et al., 1994; East et al., 2009; Al-Harthy et al., 2010; Schotsmans et al., 2011), uso de 1-MCP (Watkins, 2006; Amarante et al., 2008), uso de emulsiones de recubrimiento y tratamiento hidrotérmico (Jacomino et al., 2003; Vieira et al., 2008), uso de $\mathrm{CaCl}_{2}$ y otros tratamientos (Ramirez et al., 2005) y posición del pedúnculo durante el almacenamiento (Usushizaki et al., 1987). Estos tratamientos poscosecha utilizados para guayabas y feijoas, retrasan el proceso de maduración 
y por lo tanto, permiten conservar la calidad de los productos durante un mayor tiempo. Sin embargo, se ha demostrado que son más efectivos cuando se combinan con almacenamiento refrigerado.

\section{Almacenamiento refrigerado}

El tiempo de conservación de la feijoa en cuartos refrigerados es limitado y corresponde a aproximadamente un mes a $4^{\circ} \mathrm{C}$, seguido de $5 \mathrm{~d}$ de vida de anaquel a $20^{\circ} \mathrm{C}$ (Thorp y Klein, 1987). Valderrama et al. (2005a) indican que frutos de feijoa del 'Clon 8-4' y 'Quimba' con buen manejo poscosecha, pueden durar en buenas condiciones de calidad hasta por $16 \mathrm{~d}$. Los frutos cosechados adecuadamente e introducidos en una cadena de frío a $1,67^{\circ} \mathrm{C}$ por $21 \mathrm{~d}$, mostraron que mantienen su calidad para comercialización por 29 d más, resultado que respalda y permite pensar en su comercialización internacional por vía marítima (Valderrama et al., 2005b).

Durante el almacenamiento en frío y posterior vida útil poscosecha a temperatura ambiente, no todos los atributos de calidad cambian al mismo ritmo; el sabor es la primera característica que cambia notablemente y esto se asocia a la lenta disminución de la acidez y un pH más alto, y posiblemente una disminución de los azúcares (medida como contenido de sólidos solubles totales, SST) (Thorp y Klein, 1987; Gallego-Corrales et al, 2003; Gaddam et al, 2005; Rodríguez et al., 2006; Velho et al., 2011; Al-Harthy et al., 2010). Típicamente se reporta reducción de la firmeza y una ligera coloración amarillenta de la piel durante el almacenamiento (Akerman et al., 1993; Gallego-Corrales et al., 2003; Al-Harthy et al.,
2008) con mayores tasas de pérdida de tonalidad a temperaturas de almacenamiento superior (Amarante et al., 2008). El almacenamiento del fruto por largos periodos compromete su calidad, presentándose reducción en el sabor, el cual está asociado a reducción en la acidez y en la concentración de SST, así como en el contenido de vitamina C (Hoffmann et al., 1994).

\section{CONCLUSIONES}

El comportamiento fisiológico y las características poscosecha de los frutos (SST, ATT, firmeza, contenido de ácido ascórbico, etc.), pueden variar en una misma localidad de acuerdo con el cultivar, la edad de la planta, la época del año, las condiciones de cultivo y las condiciones de almacenamiento y manejo poscosecha de los frutos.

Los diferentes estudios poscosecha realizados indican que los frutos de feijoa presentan comportamiento típico climatérico, con elevadas tasas respiratorias y de producción de etileno y por lo tanto, necesitan refrigeración poscosecha inmediata para preservar su calidad. A medida que transcurre el proceso de maduración de los frutos, aumenta la pérdida de peso y el $\mathrm{pH}$ y disminuye la firmeza. Los resultados son contradictorios en cuanto a SST y ATT, ya que algunos autores indican que estos aumentan hasta el climaterio y luego disminuyen, mientras que otros reportan que disminuyen durante almacenamiento o permanecen constantes, comportamiento que probablemente esté influenciado por las características varietales, edad de la planta y por las condiciones climáticas y de cultivo a las que hayan estado expuestos. 


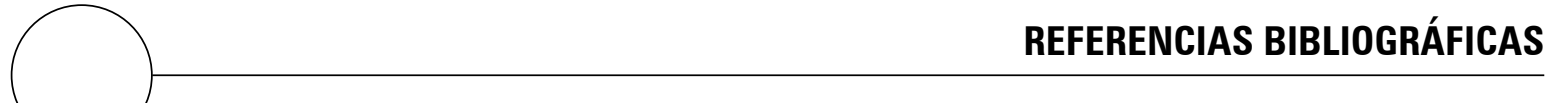

Agronet. 2012. Reportes Estadísticos. Disponible en: http://www.agronet.gov.co; consulta: septiembre de 2012.

Akerman, M., G. Zauberman y Y. Fuchs. 1993. Improvement of the keeping quality of feijoa fruit after harvest. Hassadeh 74, 55-59.

Al-Harthy, A.A.S., A.R. East, E.W. Hewett y A.J. Mawson. 2010. Controlled atmosphere storage of 'Opal Star' feijoa. Acta Hort. 876, 401-408.

Al-Harthy, A.A.S., A.J. Mawson y A.R. East. 2008. Investigations on extending shelf life of feijoa fruits with cool storage conditions. Acta Hort. 804, $255-$ 261

Amarante, C.V.T. do, C.A. Steffens, J.P.H.J. Ducroquet y A. Sasso. 2008. Qualidade de goiaba-serrana em resposta à temperatura de armazenamento e ao tratamento com 1-metilciclopropeno. Pesquisa Agropecuaria Brasileira 43(12), 1683-1689.

Bontempo, P., L. Mita, M. Miceli, A. Doto y A. Nebbioso. 2007. Feijoa sellowiana derived natural Flavone exerts anti-cancer action displaying HDAC inhibitory activities. Int. J. Biochem. Cell Biol. 39, $1902-$ 1914.

Cacioppo, O. 1982. La feijoa. Ediciones Mundi-Prensa, Madrid.

CCI (Corporación Colombia Internacional). 2008. Producción y comercialización de la feijoa en Colombia. En: http://www.cci.org.co/publicaciones; consulta: noviembre de 2010.

CCI (Corporación Colombia Internacional) 2000. La feijoa. Exótica 4(4), 17-21.

Clark, C.J., A. White, A. Woolf y K. Domijan. 2005. Can density sorting at harvest segregate mixed maturity of Feijoa? Acta Hort. 687, 93-98.

CRFG (California Rare Fruit Growers). 1996. Feijoa. En: http://www.crfg.org/pubs/ff/feijoa.html; consulta: septiembre de 2012 .

Dos Santos, K.L., N. Peroni, R.P. Guries y R.O Nodari. 2009. Traditional knowledge and management of feijoa (Acca sellowiana) in Southern Brazil. Econ. Bot. 63, 204-214.

East, A.R., X.I.T. Araya, M.L.A.T.M. Hertog, S.E. Nicholson y A.J. Mawson. 2009. The effect of controlled atmospheres on respiration and rate of quality change in "Unique" feijoa fruit. Postharvest Biol. Technol. 53(1), 66-71.

Ebrahimzadeh, M.A., F. Pourmorad y A.R. Bekhradnia. 2008. Iron chelating activity, phenol and flavonoid content of some medicinal plants from Iran. Afr. J. Biotechnol. 7, 3188-3192.

Fischer, G. 2003. Ecofisiología, crecimiento y desarrollo de la feijoa. pp. 9-26. En: Fischer, G., D. Miranda, G. Cayón y M. Mazorra, M. (eds.). 2003. Cultivo, poscosecha y exportación de la Feijoa (Acca sellowiana Berg.). Produmedios, Bogotá.

Gaddam, U.S., A.J. Mawson, W.C. Schotsmans y E.W. Hewett. 2005. Segregation of feijoa fruit using acoustic impulse response. Acta Hort. 687, 365-367.

Gallego-Corrales, S.P., C.E. Riaño-Luna y L. OrozcoGallego. 2003. Determinación del comportamiento químico y fisiológico de Feijoa sellowiana en almacenamiento. Cenicafé 54(1), 50-62.

Gálvis, J.A. 2003. Manejo de la cosecha y poscosecha de la feijoa. pp. 111-123. En: Fischer, G., D. Miranda, G. Cayón y M. Mazorra (eds.). Cultivo, poscosecha y exportación de la feijoa (Acca sellowiana Berg) Produmedios, Bogotá.

Gálvis, J.A. 2001. Estudio de comportamiento fisiológico de la Feijoa (Acca sellowiana) en condiciones de temperatura ambiente de Bogotá. Documento sin publicar. ICTA, Universidad Nacional de Colombia, Bogotá

Gálvis, J., H. Arjona, G. Fischery R. Martínez. 2002. Análisis del crecimiento y desarrollo del fruto del mango (Mangifera indica L.) variedad Van Dyke en el municipio de El Espinal. En: Memorias XXXII Congreso de la Sociedad Colombiana de Control de Malezas y Fisiología Vegetal, Santa Marta, Colombia

Giacommetti, D. y E. Lleras. 2012. Feijoa. En: FAO, Libro 09. Cultivos Andinos. La agricultura amazónica y caribeña. Mirtáceas subtropicales. En: http:// www.rlc.fao.org/es/agricultura/produ/cdrom/ contenido/libro09/Cap4_5.htm\#75; consulta: septiembre de 2012

Giovannoni, J. 2001. Molecular biology of fruit maturation and ripening. Annu. Rev. Plant Physiol. Plant Mol. Biol. 52, 725-749.

Hernández, M.S., O. Martínez y J.P. Fernández-Truji1lo. 2007. Behavior of Arazá (Eugenia stipitata Mc Vaugh) fruit quality traits during growth, development and ripening. Sci. Hortic. 111, 220-227. 
Herrera, A. y M. Quicazán. 2000. Caracterización del fruto de Feijoa (Acca sellowiana B.) Documento sin publicar. Instituto de Ciencia y Tecnología de Alimentos, Universidad Nacional de Colombia, Bogotá.

Herrero, A. y A. Guardia. 1992. Conservación de frutas. Manual Técnico. Ediciones Mundi Prensa, Madrid. pp. $161-214$

Hoffmann, A., J.C. Nachtigal, R.A. Kluge y A.B. Bilhalva. 1994. Influência da temperatura e do polietileno no armazenamento de frutos de goiabeira serrana (Feijoa sellowiana Berg.). Sci. Agric. 51(3), 563-68.

Jacomino, A.P., R. Martínez Ojeda, R.A. Kluge y J.A. Scarpare Filho. 2003. Conservação de goiabas tratadas com emulsões de cera de carnauba. Rev. Bras. Frutic. 25(3), 401-405.

Kader, A.A. 1992. Postharvest biology and technology: An overview. pp. 15-20. En: Postharvest technology of horticultural crops. University of California. Division of Agriculture and Natural Resources. Davis, CA.

Kader, A.A. 1996. Regulation of fruit physiology by controlled / modified atmosphere. Acta Hort. 398, 59-69.

Kays, S. 1997. Postharvest physiology of perishable plant products. Exxon Press, Athens, GA.

Kays, S.J. y R.E. Paull. 2004. Postharvest biology. Exxon Press, Athens, G.A.

Manabe, M. y Y. Inobe. 2005. Suppressing effects of Feijoa sellowiana Berg (Feijoa) on cytokine secretion by intestinal epithelium. Food Sci. Technol. Res. 11, 71-76.

Mercado-Silva, E., P.B. Bautista y M.A. Garcia-Velasco. 1998. Fruit development, harvest index and ripening changes of guavas produced in Central Mexico. Postharvest Biol. Technol. 13(2), 143-150.

Nakashima, H. 2001. Biological activity of feijoa peel extracts. Occasional Papers Kagoshima University Research Center for the Pacific Islands, Korimoto, Japón.

Osterloh, A., G. Ebert, W.-H. Held, H. Schulz y E. Urban. 1996. Lagerung von Obst und Südfrüchten. Verlag Eugen Ulmer, Stuttgar, Alemania.

Pantástico, E.B., A. Mattoo y T. Murata. 1984. Fisiología de la postrecolección, manejo y utilización de frutas tropicales y subtropicales. Compañía Editorial Continental, México, D.F.

Parra-Coronado, A. y J.E. Hernández-Hernández. 2008. Fisiología postcosecha de frutas y hortalizas. $4^{\mathrm{a}} \mathrm{ed}$.
Facultad de Ingeniería, Universidad Nacional de Colombia, Bogotá.

Perea, M., G. Fischer y D. Miranda. 2010. Feijoa. Acca sellowiana Berg. pp. 330-349. En: Perea, M., L.P. Matallana y A. Tirado. 2010. Biotecnología aplicada al mejoramientodeloscultivos defrutastropicales. Editorial Universidad Nacional de Colombia, Bogotá

Quintero, O.C. 2012. Feijoa (Acca sellowiana Berg). pp. 443-473. En: Fischer, G. (ed.). 2012. Manual para el cultivo de frutales en el trópico. Produmedios, Bogotá.

Ramírez, J.M., J.A. Galvis y G. Fischer. 2005. Maduración poscosecha de la feijoa (Acca sellowiana Berg) tratada con $\mathrm{CaCl}_{2}$ en tres temperaturas de almacenamiento. Agron. Colomb. 23(1), 117-127.

Rivas, A. 2004. El cultivo de la feijoa (Feijoa sellowiana Berg). Facultad de Ciencias Agropecuarias, Universidad de Caldas, Manizales, Colombia.

Rodríguez, M., A.E. Arjona y J.A. Galvis. 2006. Maduración del fruto de feijoa (Acca sellowiana Berg) en los clones 41 (Quimba) y 8-4 a temperatura ambiente en condiciones de la Sabana de Bogotá. Agron. Colomb. 24(1), 68-76.

Schotsmans, W.C., G. Thorp y A.B. Woolf. 2011. Feijoa (Acca sellowiana [Berg] Burret). pp. 115-133. En: Yahia, E.M. (ed.) Postharvest biology and technology of tropical and subtropical fruits. Vol. 3. Woodhead Publishing, Oxford, U.K.

Schuman, M. y P. Lüdders. 1992. Cultivation of feijoa: Possibilities and limits in Colombia. Erwerbsobstbau 34(4), 110-112.

Seymour, G., J. Taylory G. Tucker (eds.). 1993. Biochemistry of fruit ripening. Chapman and Hall, London.

Solarte, M.E., M.S. Hernández, A.L. Morales, J.P. Fernández y L.M. Melgarejo. 2010. Caracterización fisiológica y bioquímica del fruto de guayaba durante la maduración. pp. 85-119. En: Morales, A.L. y L.M. Melgarejo (eds.). 2010. Desarrollo de productos funcionales promisorios a partir de la guayaba (Psidium guajava L.) para el fortalecimiento de la cadena productiva. Facultad de Ciencias, Universidad Nacional de Colombia, Bogotá.

Thorp, G. y J.D. Klein. 1987. Export feijoas: post-harvest handling and storage techniques to maintain optimum fruit quality. Orchardist NZ, 164-166.

Thorp, G. y R. Bieleski (eds.). 2002. Feijoas: origins, cultivars and uses. David Bateman Ltd., Auckland, New Zealand.

Usushizaki, S., N. Nagashima, Y.; Takeda y Y. Ota. 1987. Effect of postures of vegetables and fruits in stora- 
ge on keeping postharvest freshness. Jpn. Agr. Res. Quart. 20(3), 196-201.

Uyabán, D. y N. Téllez. 2009. Características físico-químicas en diferentes partes del fruto de feijoa (Acca sellowiana Berg) y de curuba (Passiflora mollissima Bailey). Trabajo de grado. Facultad de Agronomía, Universidad Nacional de Colombia, Bogotá.

Valderrama, J.K., G. Fischer y M.S. Serrano. 2005a. Fisiología poscosecha en frutos de dos cultivares de feijoa (Acca sellowiana [O. Berg] Burret) sometidos a un tratamiento cuarentenario de frío. Agron. Colomb. 23(2), 276-282.

Valderrama, J.K., M.S. Serrano y G. Fischer. 2005b. Mortalidad de larvas de Anastrepha fraterculus (Wiedemann) (Diptera: Tehritidae) en frutos de feijoa (Acca sellowiana [O. Berg] Burret) sometidos a un tratamiento cuarentenario de frío. Rev. Colomb. Entomol. 31(2), 171-176.

Vela, P., C. Salinero, P. Piñon y M.J. Sainz. 2009. Características del fruto de Acca sellowiana cultivada en Galicia. En: http://www.efa-dip.org/comun/ publicaciones/comunicaciones/2009/feijoa\%20(logrono).pdf; consulta: marzo de 2013.

Velho, A.C., Do Amarante, C.V.T, L.C. Argenta y C.A. Steffensl. 2011. Influência da temperatura de ar- mazenamento na qualidade pós-colheita de goiabas serranas. Rev. Bras. Frutic. 33(1), 14-20.

Vieira, S.M.J., S.M. Couto, P.C. Corrêa, A.E.O. dos Santos, P.R. Cecom, y D.J.P. da Silva. 2008. Características físicas de goiabas (Psidium guajava L.) submetidas a tratamento hidrotérmico. Rev. Bras. Eng. Agríc. Ambiental 12(4), 408-414.

Vuotto, M.L., A. Basile, V. Moscatiello, P. de Sole, R. Castaldo-Cobianchi, E. Laghi y M.T.L. Lelpo. 2000. Antimicrobial and antioxidant activities of Feijoa sellowiana fruit. Int. J. Antimicrobial Agents 13, 197-201.

Watkins, C.B. 2006. The use of 1-methylcyclopropene (1-MCP) on fruits and vegetables. Biotechnol. Adv. 24, 389-409.

Wills, R., B. McGlasson, D. Graham y D. Joyce. 1998. Postharvest: An introduction to the physiology and handling of fruit, vegetables and ornamentals. CABI Publishing. Wallingford, U.K.

Wilson, P.G., M.M. O'Brien, P.A. Gadek y C.J. Quinn. 2001. Myrtaceae revisited: a reassessment of infrafamilial groups. Amer. J. Bot. 88(11), 2013 2025. 